\title{
USE OF CONOIDAL CURVES IN CORNEAL CONTACT LENSES*
}

\author{
BY \\ MONTAGUE RUBEN \\ Moorfields Eye Hospital, High Holborn Branch, London
}

THE development of corneal lenses in recent years has been directed towards more accurate fitting of the cornea (Dallos, 1964; Ellerbrock, 1963; Girard, 1964; Morrison, Kaufman, and Cerulli, 1964).

It is not the purpose of this paper to indicate the many clinical reasons why a perfect fit cannot be achieved (Ruben, 1964). Whether contact lenses of small or large diameters are used, 2 or 10 curves, the object of the fitter has been to control the curves relative to the cornea. This problem has resolved itself into the fitting of a central and peripheral zone of the cornea. This may be based on keratometry and/ or trial lenses. The blending of several peripheral back curves to form a "paraboloidal' curve provides a smooth transition from optical zone to lens edge. The type of paraboloidal curve formed by blending many curves of small widths is not controlled by the present blending techniques. It is necessary, therefore, to have a paraboloidal type curve which can be controlled by definition and be reproduced faithfully by manufacturing processes. Such a lens could be produced accurately from a prescription. Studies of corneal contours and anterior segment scleral contours by many methods, including shadowgrams of eye impressions (Bonnet, Massin, and Rapilly, 1964; Dallos, 1933; Mandell, 1965; Ruben, 1964), show that the apical curves of the cornea are not concentric with the peripheral curves. Therefore, the peripheral curves of the cornea, or for that matter of the sclera, can only be approximately fitted if concentric curves are used (Fig. 3, below). Fortunately, the pre-corneal tear layer, the softness and elasticity of the cornea, and the thinness of the contact lens and its flexibility, together with moulding movements of the lids and surface tension forces, permit a margin of tolerance in fitting techniques. Therefore, once initial tolerance is achieved by the cornea and lid, good wearing times are obtained with many types of corneal contact lenses, but opinion differs as to the design of peripheral curves (Bayshore, 1963; Wesley, 1963).

A simulated paraboloidal curve as shown in Fig. 1 (opposite) has been used in preformed scleral lens design (Forknall, 1959; McKellen, 1948). This eccentric curve from the central curve to the edge can be cut on the lathe (Benett, 1965). The radius of this curve is determined by the degree of flattening required to produce the most peripheral curve (excluding the edge curve). The curve has its centre eccentric to the optical zone centre, so that the transition from E to D (Fig. 1) is continuous and simulates a paraboloidal curve. It is, in fact, quasi-conoidal and therefore like that of the cornea. Such curves would be important in fitting conoidalshaped corneae as well as normal ones.

\footnotetext{
* Received for publication July 16, 1965.
} 


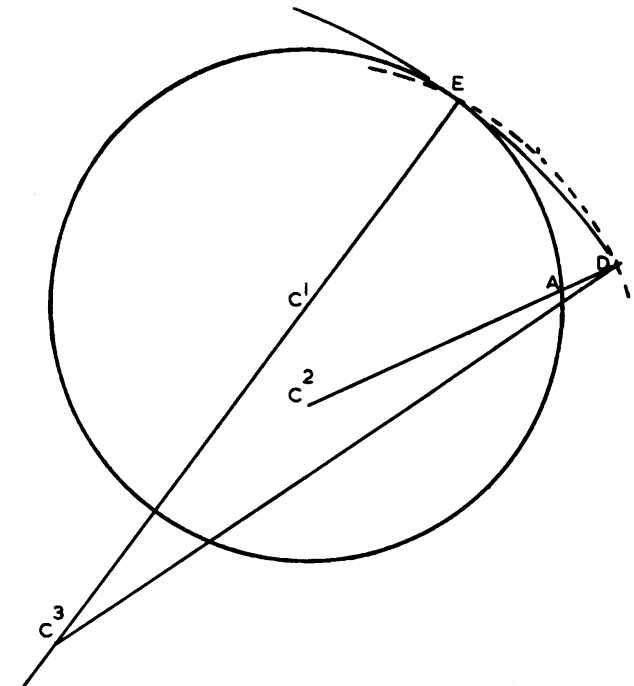

Fig. 1.-Contralateral offset peripheral curve, showing the back optic radius curvature with centre at $\mathrm{Cl}$ and secondary peripheral curve (interrupted line) with centre at $\mathbf{C 2}$. $\quad \mathbf{C 1}$ and $\mathrm{C} 2$ are monaxial. Continuous line ED is conoidal curve by decentring peripheral curve to C3. Note tangential nature of this curve with back optic radius curve at $E$ and simulation of paraboloidal curve. $\quad \mathbf{A D}=$ flattening factor.

It was intended to use the offset peripheral curve in corneal lenses in two forms: to offset the curve on the same side of the central axis (see Fig. 2) and to offset on the opposite side (Fig. 1), the flattening factor required from edge of optic curve to edge of the lens being identical. The homolateral (same side) offset curves must be blended by polishing in the area of transition, but with normal corneal contours this is only very little. The homolateral offset curves used in this present clinical trial were cut to the same radius as the centre back curve. This has the advantage of providing a peripheral fitting curve without a change in focal power. This can be of importance to avoid aberrations when wide peripheral curves are used. The contralateral offset curves will simulate the corneal contour more exactly but produce a peripheral optical power change of a plus nature if required. This may be of importance in bifocal corneal lens design.

In the present trial, a flattening factor of $0.75 \mathrm{~mm}$. for a lens diameter not exceeding $9.5 \mathrm{~mm}$. was found to be most satisfactory for normal ranges of corneae. All curves were cut on a lathe.

A further extension of this idea at present under trial is to use more than one curve of this type should larger lenses be required. Indeed scleral lenses might be made in this way (Fig. 3, overleaf). Such a lens is planned on the drawing board, using the shadowgram outline as a basis. It would even be possible to employ toroidal contours by this method.

\section{Fitting Procedure}

The apical zone of the cornea according to Soper varies in diameter, in position, and in contour of parameter, and can be estimated by topographical keratometry. The average longitudinal diameter is about $6 \mathrm{~mm}$. and most corneal contour lenses of the tri-curve type 


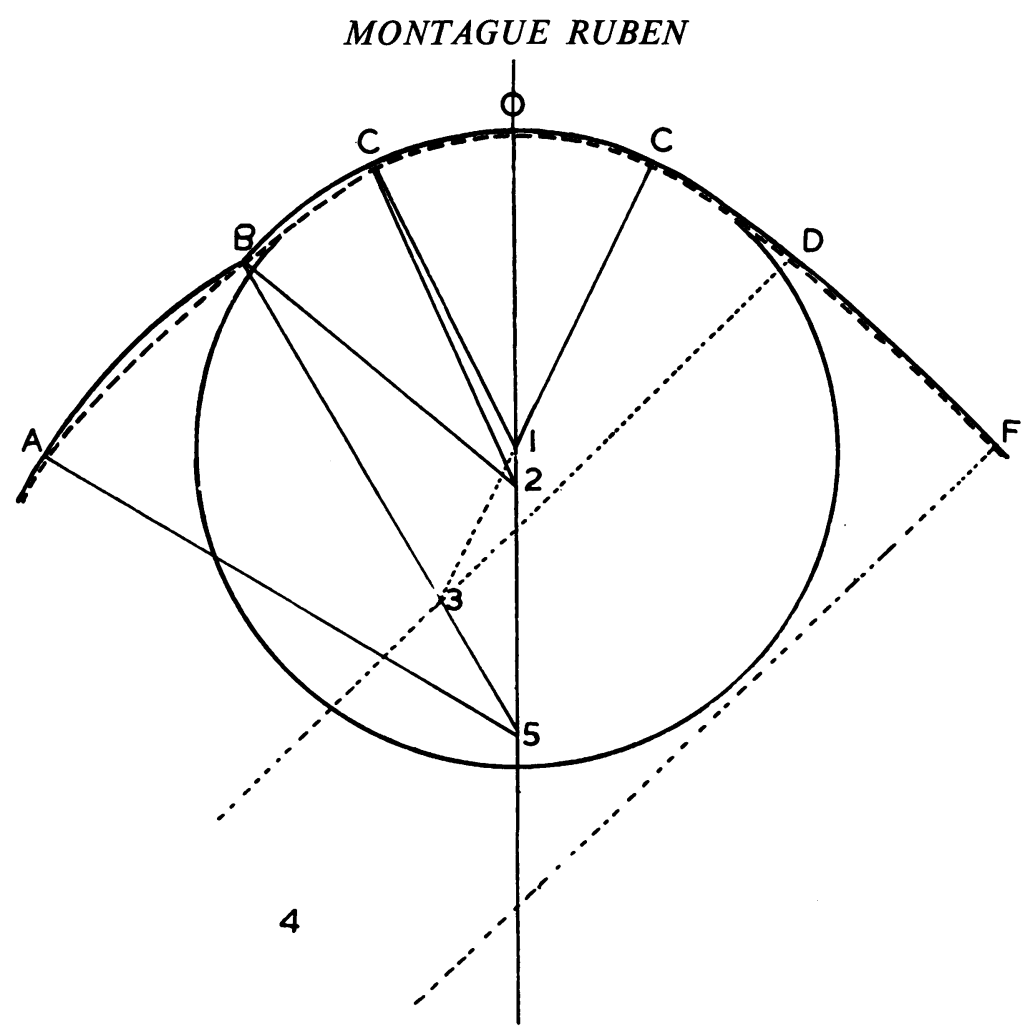

FIG. 3.-Contralateral offset peripheral curve, showing alignment of monaxial centre curves compared with eccentric curves (paraboloidal simulation curves). AOF (interrupted line) represents outline of anterior segment of eye taken from shadowgram. Centre 1 gives back optic radius of curvature arc COC; Centre 2 first peripheral curve arc BC; Centre 5 second peripheral curve arc BA. Eccentric Centres 3 and 4 for right side of diagram to give arcs $\mathrm{CD}$ and DF. Arcs $\mathrm{CB}$ and $\mathrm{AB}$ are not in alignment with normal eye contour but $\mathrm{CD}$ and $\mathrm{DF}$ are.

have an apical back curve diameter of $6.5 \mathrm{~mm}$. (Girard, 1964). The topographical methods of recording keratometry are not easy and certainly time-consuming. The alternative for routine practice is to have fitting sets of lenses with varying diameters of the back optic diameter (de Lesdain, 1964). I use four back optic apertures (diameter 5·5, 6·7, 7·5, and $9 \mathrm{~mm}$.) having respectively overall diameters of $8 \cdot 5,9 \cdot 5,10 \cdot 5$, and $12 \mathrm{~mm}$. This permits assessment of the central zone fit (Biri, 1964; de Lesdain, 1964; Dunn, 1959). It is possible to control apical clearance and to use curves conforming to those of the cornea by this method (Fig. 4, opposite). I have even been able, using the 10 and $12 \mathrm{~mm}$. lenses, to obtain apical clearances over highly astigmatic corneae without the use of toric curves. This method of fitting is therefore not dissimilar to accepted scleral fitting techniques. The trial lenses have a power of -4 dioptres, since this permits a spectacle addition of $+4 \mathrm{D}$ to $-4 \mathrm{D}$ without effective power calculations. The flattening factor of the simulated paraboloidal curve is increased to $1 \mathrm{~mm}$. for the larger diameter lenses. In high refractive errors, it is important to assess the fit by using this range of fitting lenses, preferably combined with a high-power trial set. In this way inaccuracies in final power and fit are avoided.

\section{Conclusion}

Four hundred eyes have been fitted with homolateral offset curves and two hundred with contralateral offset curves. Such 'paraboloidal' curves are more easily prescribed and more accurately dispensed than the multicurve lenses hitherto used. The patients fitted included approximately one-third with normal refractive errors, one- 


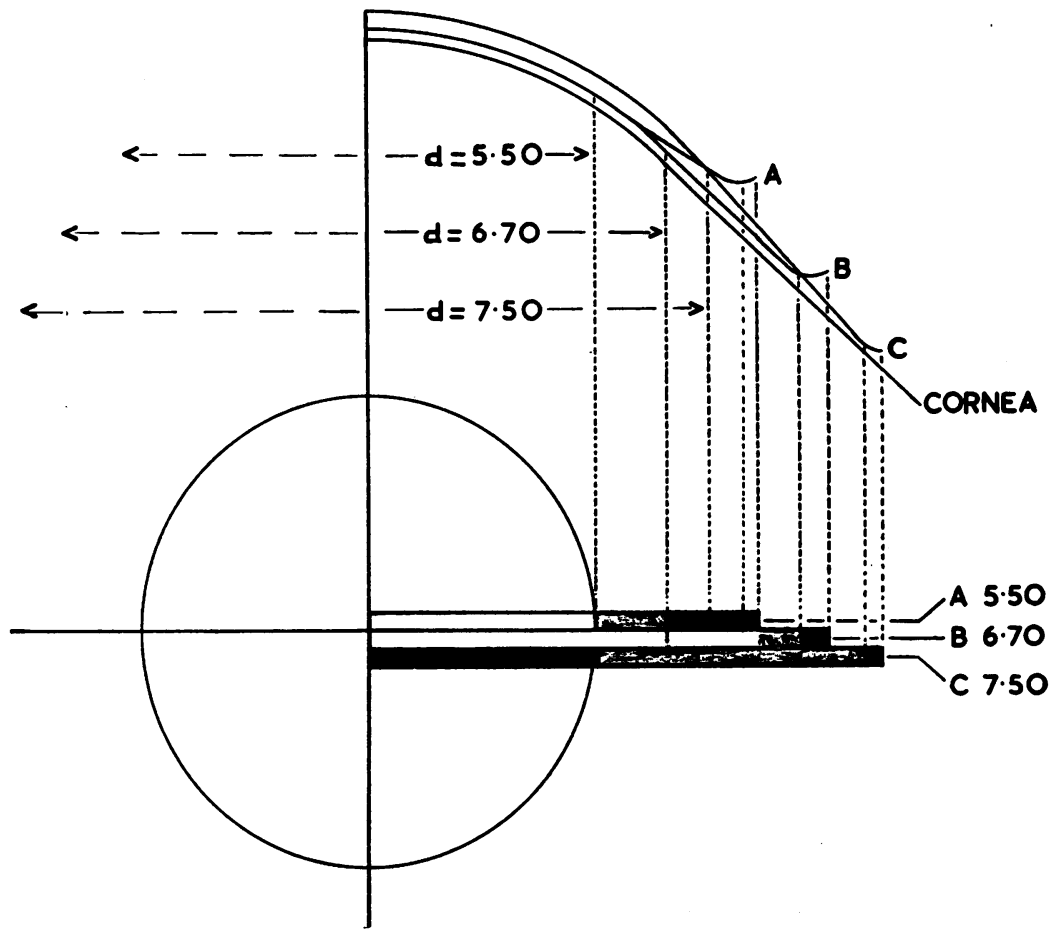

Fig. 4.-Three trial lenses in position for a given corneal curve, where corneal cap radius of curvature is exactly that of three trial lenses, A, B, C. Projected strips for comparison of fluorescein distribution in the corneo-lens space. Trial lens A, back optic diameter 5.50 is in contact over central area. Lens B is in contact almost to edge curves. Lens $\mathbf{C}$ shows clearance over whole of central area. Therefore, cap diameter lies between 5.50 and $6.50 \mathrm{~mm}$. and nearest to $6.50 \mathrm{~mm}$. in the flattest meridian shown.

third with aphakic eyes, and one-third with high myopia. No abnormalities in follow-up examinations were noted as compared with patients fitted with orthodox corneal lenses of bi- or tri-curve design.

I should like to thank Mr. Frederick Ridley for his helpful reading of this paper, and Miss M. Ryder and Mr. T. Tarrant (Medical Illustration Department, Institute of Ophthalmology) for assistance with the diagrams.

All homolateral offset curves and lathe setting were done by G. Nissel and Co. Ltd., and some contralateral lenses were made by Omega Ltd., to whom I am exceedingly grateful. The lathes were manufactured by $\mathbf{G}$. Nissel.

\section{REFERENCES}

Bayshore, C. A. (1963). Amer. J. Optom., 40, 351.

BenNett, A. G. Personal communication to G. Nissel.

BIRI, H. (1964). “Contact Lens Congress, 1964. Royaumont" ("Lentilles optiques rationelles").

Bonnet, Massin, and Rapilly (1964). Bull. Soc. Ophtal. Fr., 64, 487.

Dallos, J. (1933). Klin. Mbl. Augenheilk., 91, 640. (1964). Brit. J. Ophthal., 48, 510.

DunN, G. M. (1959). Optician, 138, 501.

Ellerbrock, V. J. (1963). Amer. J. Optom., 40, 439.

Forknall, A. J. (1959). Brit. J. Ophthal., 16, 96.

GiRARD, L. (1964). “Corneal Contact Lenses". Mosby, St. Louis.

LESDAIN, F. DE (1964). "Les lentilles cornéennes à courbures physiologiques". Masson, Paris.

Mandell, R. (1965). "Contact Lens Practice”, pp. 46-48. Thomas, Springfield, Ill.

McKellen, S. D. (1948). Optician, 116, 497.

Morrison, R. J., Kaufman, R. J., and Cerulli, E. (1964). Amer. J. Optom., 41, 688.

RuBEN, M. (1964). Trans. ophthal. Soc. U.K., 84, 15.

WesLeY, N. K. (1963). Amer. J. Optom., 40, 150. 